\title{
Immediate Umbilical Reconstruction after a Mohs Micrographic Surgery for Primary Cutaneous Adenoid Cystic Carcinoma Arising in the Umbilicus
}

\author{
Jee Eun Kim, Mi Ryung Roh, Kee-Yang Chung ${ }^{1}$ \\ Department of Dermatology, Gangnam Severance Hospital, Cutaneous Biology Research Institute, Yonsei University College of Medicine, \\ ${ }^{1}$ Department of Dermatology, Severance Hospital, Cutaneous Biology Research Institute, Yonsei University College of Medicine, Seoul, Korea
}

\begin{abstract}
Adenoid cystic carcinoma (ACC) is a malignant neoplasm of glands commonly occurs in salivary glands. Primary cutaneous adenoid cystic carcinoma (PCACC) is a rare form of ACC that primarily presents on the skin. Herein, we represent a rare case of PCACC occurred in the umbilicus in a 66 -year-old Korean male patient. The patient visited our center with erythematous indurated patch on the umbilicus diagnosed as ACC by incisional biopsy at another center. The diagnosis of PCACC was confirmed by additional histopathologic examination and imaging study. We proceeded Mohs micrographic surgery and reconstructed umbilicus with tacked purse string suture. Local recurrence and distant metastasis were not observed during 30-month follow-up. We report this rare case of PCACC on the umbilicus so that dermatologist can aware of the rare disease. Furthermore, we recommend MMS and tacked purse string suture as effective methods for treatment of PCACC and immediate umbilical reconstruction. (Ann Dermatol 31(6) 669 672, 2019)
\end{abstract}

\section{-Keywords-}

Immediate umbilical reconstruction, Mohs micrographic

Received February 26, 2018, Revised August 24, 2018, Accepted for publication October 17, 2018

Corresponding author: Kee-Yang Chung, Department of Dermatology, Severance Hospital, Cutaneous Biology Research Institute, Yonsei University College of Medicine, 50-1 Yonsei-ro, Seodaemun-gu, Seoul 03722, Korea. Tel: 82-2-2228-2080, Fax: 82-2-393-9157, E-mail: kychung@yuhs.ac ORCID: https://orcid.org/0000-0003-3257-0297

This is an Open Access article distributed under the terms of the Creative Commons Attribution Non-Commercial License (http://creativecommons. org/licenses/by-nc/4.0) which permits unrestricted non-commercial use, distribution, and reproduction in any medium, provided the original work is properly cited.

Copyright (c) The Korean Dermatological Association and The Korean Society for Investigative Dermatology surgery, Primary cutaneous adenoid cystic carcinoma

\section{INTRODUCTION}

Adenoid cystic carcinoma (ACC) is a malignant neoplasm of glands commonly occurs in salivary glands. Primary cutaneous adenoid cystic carcinoma (PCACC) is a rare form of ACC that primarily presents on the skin showing indolent course with low rate of distant metastasis. To date, less than 70 cases have been reported in the English literature. Herein, we report a case of PCACC of the umbilicus. To the best of author's knowledge, this is he first reported case of PCACC in the umbilicus.

The patient was diagnosed as PCACC upon histopathological examination and the lesion was removed by Mohs micrographic surgery (MMS). Since umbilicus is an aesthetically important body structure located at the center of the abdomen, the sacrificed umbilicus in the process of the surgery was reconstructed with special technique of purse string sutures.

\section{CASE REPORT}

A 66-year-old Korean male patient presented with an illdefined erythematous indurated patch on the umbilicus (Fig. 1A). The patient had not received any treatment for 1 year as the lesion was asymptomatic. Recently, the patient was referred to our center after an incisional biopsy. The tumor was cleared after three stages of MMS. We received the patient's consent form about publishing all photographic materials.

Histopathologic examination revealed basaloid tumor cells forming nests and cords in the dermis and subcutaneous 

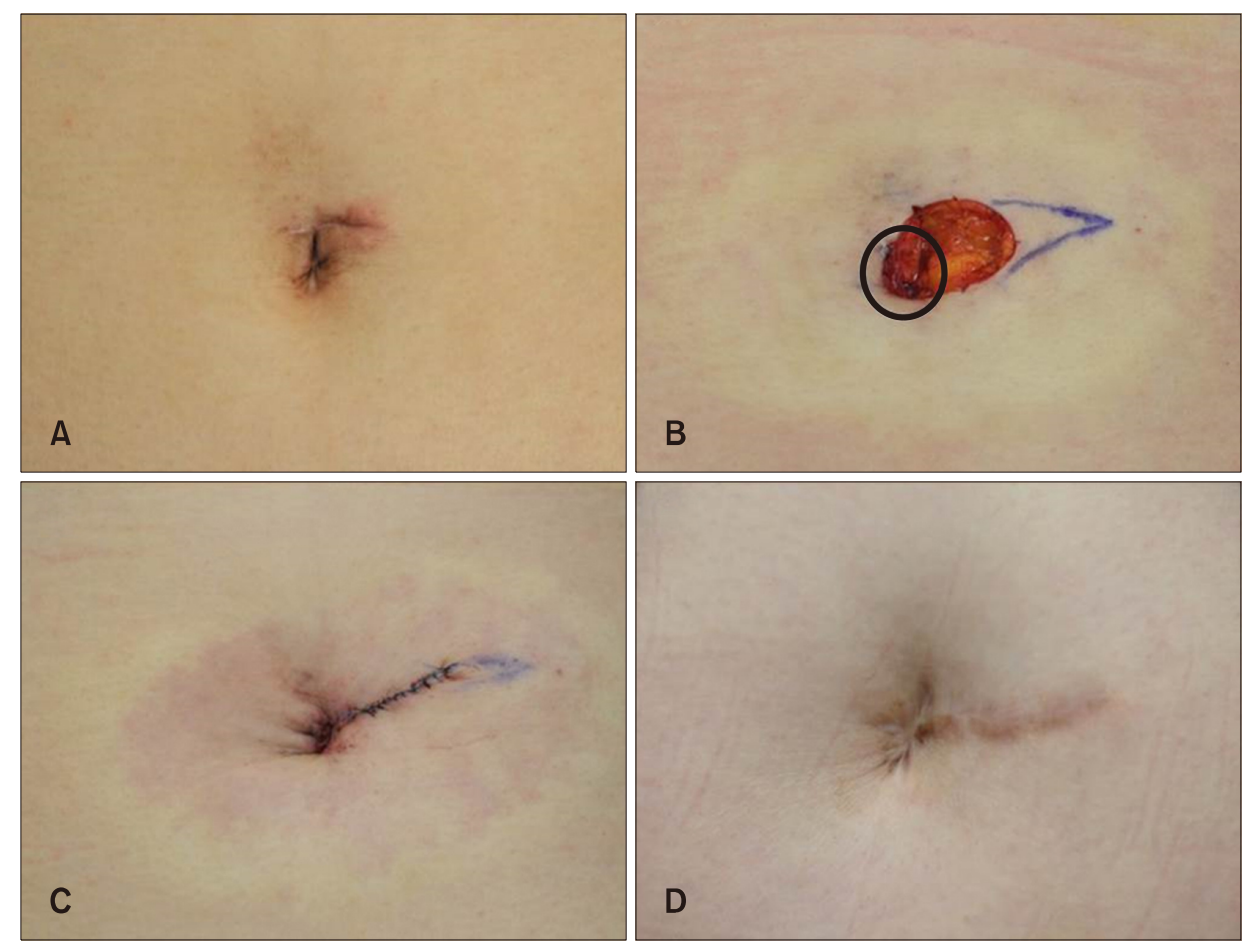

Fig. 1. (A) An ill-defined erythematous nodule mainly on the left upper side of the umbilicus. (B) Surgical defect after 3 stages of Mohs micrographic surgery. Black circle denotes the location of the umbilicus. (C) Reconstruction of umbilicus in a centralized defect with tacked purse string sutures. (D) The umbilicus was well maintained after 20 months of followup.
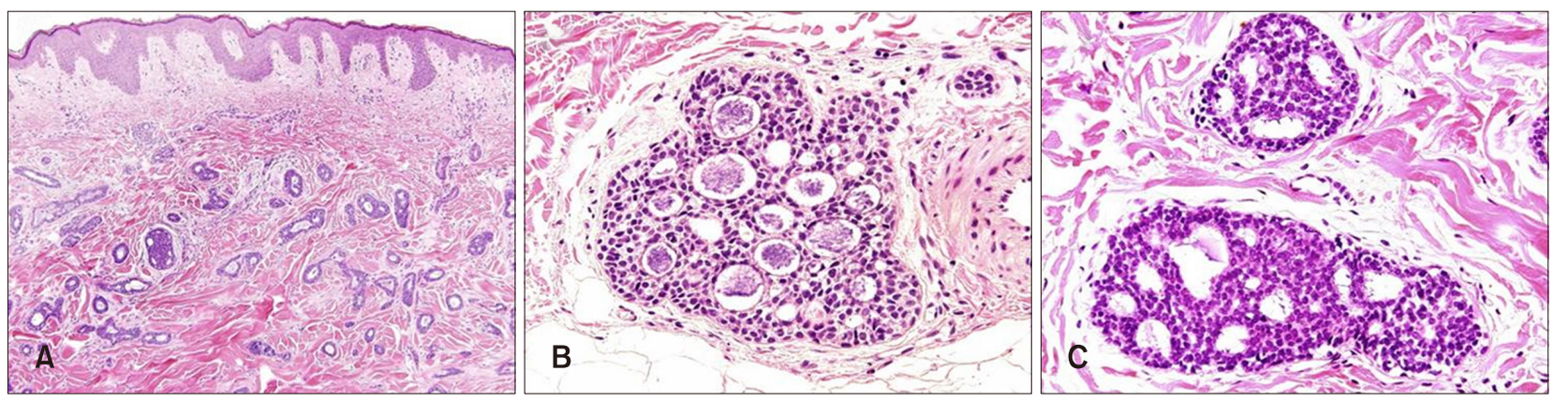

Fig. 2. Histopathologic features of the excised tumor. (A) Basaloid tumor cells forming nests and cords in the dermis and subcutaneous tissue without connection to the epidermis $(\mathrm{H} \& \mathrm{E}$, original magnification $\times 100)$. (B) Cribriform or tubular structure of the basaloid cells with abundant mucin inside $(\mathrm{H} \& \mathrm{E}, \times 400)$. (C) Tumor cells had hyperchromatic nuclei but did not demonstrate distinct cellular atypia $(\mathrm{H} \& \mathrm{E}, \times 400)$.

tissue (Fig. 2A). The tumor showed cribriform or tubular structure without connection to the epidermis. Abundant mucin was observed inside the tubular structure (Fig. 2B). Tumor cells had hyperchromatic nuclei but did not demonstrate distinct cellular atypia (Fig. 2C). Immunohistochemical study against receptor tyrosine kinase (cKIT) and epithelial membrane antigen (EMA) showed strong positive for tumor cells. The tumor was diagnosed as ACC upon histopathologic examination and immunohistochemical study. Physical examination and whole-body positron emission tomography fused with computed tomography (PET/CT) performed to exclude the possibility of metastasis from other original lesion showed no evidence of additional lesion. Finally, the patient was diagnosed as PCACC.

In the process of removing the tumor, the umbilicus was completely eliminated with an asymmetrical defect that extended into the left upper quadrant, which required elaborate reconstruction. The size of the postoperative defect was $2.0 \times 1.8 \mathrm{~cm}$ (Fig. 1B). An elliptical repair was done at the left side of the defect to close the paramedian defect as well as to remove the dogear. To close the remaining central defect and to recreate the concavity of the umbilicus, about $0.5 \mathrm{~cm}$ of the soft tissue was removed from the surgical margin, deeper layered purse string sutures were placed to adjust the size of the umbilicus, and the superficial purse string suture involving the dermal layer was 


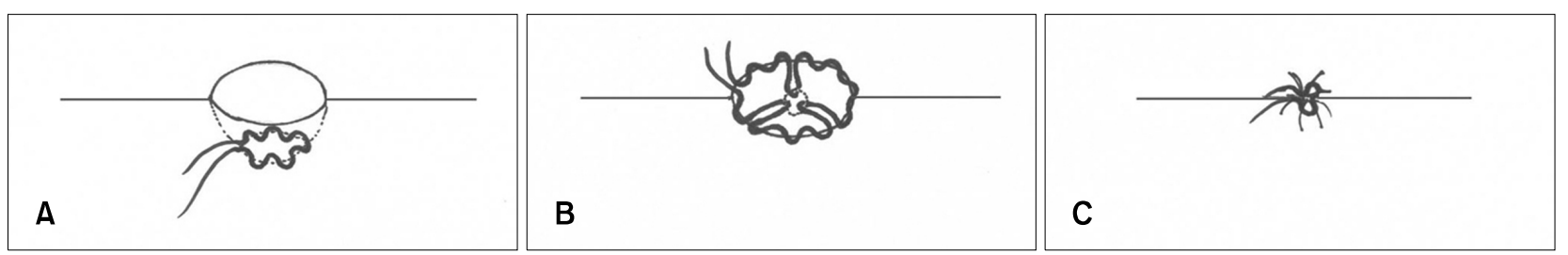

Fig. 3. (A) Deeper layered purse string sutures were placed to adjust the size of the umbilicus. (B) The superficial purse string suture involving the dermal layer was tacked at 3 areas at 12, 4, and 8 o'clock directions to the underlying linea alba. (C) Concave and normal looking umbilicus was recreated after tightening the purse string suture.

tacked at 3 areas at 12, 4, and $8 \mathrm{o}^{\prime}$ clock directions to the underlying linea alba (Fig. 3). Concave structure of the umbilicus was recreated at the center of the abdomen (Fig. 1C). Follow-up at six months showed a well-healed surgical defect with recreated concave and normal looking umbilicus and its shape was well maintained at 20-month follow-up (Fig. 1D).

The patient showed no local recurrence or distant metastasis at 30-month follow-up.

\section{DISCUSSION}

ACC is a rare tumor which occurs most commonly in the head and neck, especially in the major and minor salivary glands. It can arise from a variety of primary sites including the breast, trachea, external auditory canal, uterus and prostate $^{1}$. It also occurs on the skin primarily or metastatically. Cutaneous ACC usually presents as a solitary, flesh-colored or erythematous, ill-defined, slow-growing mass ${ }^{1}$. Patients are usually asymptomatic or may present with tenderness. PCACC is diagnosed upon histopathologic examination, which shows basaloid cells with hyperchromatic nuclei arranged in a tubular or cribriform architecture with abundant mucin in the cystic spaces ${ }^{2}$. cKIT and EMA are known to be strongly reactive in the $\mathrm{PCACC}^{2}$. For confirming the diagnosis of PCACC, since PCACC is not distinguishable from other ACCs by histopathologic examination, the possibility of metastasis should be excluded by imaging studies. Our case was confirmed as PCACC after excluding the possibility of metastatic lesion with whole-body PET/CT.

The standard treatment for PCACC is wide local excision. PCACC shows high tendency of local recurrence, though overall disease course is indolent with low rate of distant metastasis ${ }^{2}$. Thus, it is important to secure enough safety margin to reduce the opportunity of local recurrence. Recently, MMS is considered as the adequate treatment for PCACC, as MMS shows better outcome with lower rate of local recurrence and minimal skin defect in other skin cancers, such as squamous cell carcinoma or basal cell carcinoma. Among reported 7 cases of cutaneous ACC treated with MMS, there has been no local recurrence with a follow-up range of 10 to 28 months $^{3}$. Although significant conclusion cannot be made due to the short observation period and the insufficient number of cases, MMS is well worth to be considered as the treatment for PCACC. Our case was treated with MMS and did not show recurrence at 30-month follow-up.

In the process of abdominal surgery including urachal cyst repair, umbilical herniorrhaphy and tumor excision, it is often unavoidable to sacrifice the umbilicus. Various methods for umbilical reconstruction have been developed including an inverted C-V flap ${ }^{4}$ or a lunchbox approach ${ }^{5}$. However, these methods leave extensive scars around the reconstructed umbilicus and the three-dimensional structure of the reconstructed umbilicus may become flattened with passing of time. The purse string suture has been reported to be a method that allows immediate reconstruction of umbilicus with minimizing the scars ${ }^{6-8}$. This method also allows formation of the three-dimensional structure of the umbilicus and is suitable for long-term maintenance of the umbilical shape. In addition, in some cases of tumor surgery involving the umbilicus, as in our case, the surgeon has to deal with an asymmetric skin defect. In this situation, initial procedure to centralize the defect is necessary. After centralization of the defect, defatting of the surgical margin followed by double-layered purse string suture with the superficial suture being tacked down at three locations will successfully recreate a cosmetically acceptable umbilicus.

In summary, we reported a case of a rare cutaneous tumor, PCACC occurred on the umbilicus. The histopathologic features and the results of immunohistochemical study were suitable for diagnosis of ACC. The exclusion of other possible origin for the lesion was made before the final diagnosis of PCACC. The lesion was removed by MMS and the umbilicus was reconstructed with tacked purse string suture. The patient is maintaining three-dimensional 
umbilicus with no local recurrence or distant metastasis for 30-month follow-up. In our experience, MMS and tacked purse string suture were effective methods for treatment of PCACC and immediate umbilical reconstruction.

\section{CONFLICTS OF INTEREST}

The authors declare no conflicts of interest.

\section{ORCID}

Jee Eun Kim, https://orcid.org/0000-0003-0795-5742

Mi Ryung Roh, https://orcid.org/0000-0002-6285-2490

Kee-Yang Chung, https://orcid.org/0000-0003-3257-0297

\section{REFERENCES}

1. Ramakrishnan R, Chaudhry IH, Ramdial P, Lazar AJ, McMenamin ME, Kazakov D, et al. Primary cutaneous adenoid cystic carcinoma: a clinicopathologic and immunohistochemical study of 27 cases. Am J Surg Pathol 2013;37:
1603-1611.

2. Naylor E, Sarkar P, Perlis CS, Giri D, Gnepp DR, RobinsonBostom L. Primary cutaneous adenoid cystic carcinoma. J Am Acad Dermatol 2008;58:636-641.

3. Xu YG, Hinshaw M, Longley BJ, Ilyas H, Snow SN. Cutaneous adenoid cystic carcinoma with perineural invasion treated by mohs micrographic surgery-a case report with literature review. J Oncol 2010;2010:469049.

4. Shinohara $\mathrm{H}$, Matsuo $\mathrm{K}$, Kikuchi N. Umbilical reconstruction with an inverted C-V flap. Plast Reconstr Surg 2000;105: 703-705.

5. Onishi K, Yang YL, Maruyama Y. A new lunch box-type method in umbilical reconstruction. Ann Plast Surg 1995. 35:654-656.

6. Bartsich SA, Schwartz MH. Purse-string method for immediate umbilical reconstruction. Plast Reconstr Surg 2003; 112:1652-1655.

7. Schoeller T, Rainer C, Wechselberger G, Piza-Katzer H. Immediate navel reconstruction after total excision: a simple three-suture technique. Surgery 2002;131:105-107.

8. Meirer R, Wechselberger G, Schoeller T. Purse-string method for immediate umbilical reconstruction. Plast Reconstr Surg 2004; 114:831-832. 